\title{
Hydrogen peroxide as regeneration-initiation signal that activates pERK to trigger planarian regeneration
}

\section{Vincent Jaenen ${ }^{1}$, Susanna Fraguas ${ }^{2}$, Karolien Bijnens ${ }^{1}$, Mireia Vaca ${ }^{2}{ }^{3}$, Tom Artois ${ }^{1}$, Rafael Romero ${ }^{2}$, Karen Smeets ${ }^{1 *}$, Francesc Cebrià ${ }^{2,4}$ *}

1

Centre for Environmental Sciences, Hasselt University, Diepenbeek, Belgium

2

Department of Genetics, Microbiology and Statistics, Faculty of Biology, University of Barcelona, Barcelona, Spain

3

Present address: Department of Experimental and Health Sciences, University Pompeu Fabra, Barcelona, Spain

4

Institute of Biomedicine of the University of Barcelona (IBUB), University of Barcelona, Barcelona, Spain

\section{*Equally contributed}

Co-Corresponding authors:

Francesc Cebrià

Department of Genetics, Microbiology and Statistics

Faculty of Biology

Av. Diagonal 643, 08028 Barcelona, Spain

fcebrias@ub.edu

Karen Smeets

Department of Biology and Geology

Faculty of Sciences

Agoralaan Building D, 3590 Diepenbeek, Belgium

karen.smeets@uhasselt.be 


\begin{abstract}
Despite the extensive research on molecular pathways controlling the process of regeneration in planarians and other regeneration models, little is known about the actual initiation signals necessary to induce regeneration. Previously the involvement of ROS, EGFR and MAPK/ERK has been demonstrated during planarian regeneration, however the exact interplay has not been yet described. By selectively interfering with major mediators in all three key parts (ROS, EGFR \& MAPK/ERK), we were able to identify amputation/wound-induced ROS, and $\mathrm{H}_{2} \mathrm{O}_{2}$ specifically, as upstream cue in activating regeneration-initiation. In addition, our results demonstrate new relationships between regeneration related ROS production and MAPK/ERK activation at early regeneration stages, as well as the involvement of the EGFR-signaling pathway. In summary, our results suggest a new and more extensive signaling model with ROS, and $\mathrm{H}_{2} \mathrm{O}_{2}$, highlighted as upstream initiationfactor and its important functions in the downstream EGFR-MAPK/ERK pathway during planarian regeneration.
\end{abstract}




\section{Introduction}

Regeneration is the fascinating phenomenon in which animals are able to repair and regrow lost or damaged tissues, structures and even the whole body ${ }^{1}$. At the cellular level, this includes proliferation, migration and differentiation, processes that need to be under a strict genetic and molecular control ${ }^{2}$. The capacity to regenerate missing or damaged parts is widely distributed across the animal kingdom ${ }^{3}$. The level of regeneration varies greatly among different species; from full body regeneration in invertebrates such as hydra and planarians, to specific organs and structures as for example limb regeneration in amphibians and heart regeneration in zebrafish ${ }^{4-6}$. Mammals such as humans, on the other hand, have very limited regenerative capabilities. Understanding how tissue development takes place in regenerative animals might provide fundamental knowledge for the further improvement of regenerative medicine.

In this context, freshwater planarians are a model with several attractive features: i) they can regenerate a whole animal from a tiny piece of their body, ii) a large part of their body consists of a population of adult pluripotent stem cells called neoblasts, with a similar transcriptional profile as compared to other invertebrate and vertebrate stem cells, and iii) they use conserved signalling pathways to regulate cell differentiation, patterning and morphogenesis. As such, Hedgehog and Wnt/ $\beta-$ catenin signaling are required to re-establish the anteroposterior axis of the animal, the BMP pathway regulates the dorsoventral axis, and the EGFR pathway is needed for proper neoblast differentiation ${ }^{7-9}$. Although several studies have uncovered a pivotal role of these, and other, signaling pathways during planarian regeneration, little is known about what upstream signals activate them to trigger a regenerative answer after an amputation. Recently, Owlarn and colleagues reported that a planarian extracellular signal-regulated protein kinase (ERK) was strongly activated in a stem cell-independent manner, just minutes after amputation. By inhibiting protein synthesis using cycloheximide, they showed that ERK activation was triggered by injury signals that did not originate from newly synthesized proteins. Therefore, ERK activation by a still unknown factor, appears to be the most upstream initiator of planarian regeneration ${ }^{10}$. In recent years, many in vitro as well as in vivo studies put forward reactive oxygen species (ROS) as upstream signaling molecules of regeneration ${ }^{11-13} \cdot \mathrm{H}_{2} \mathrm{O}_{2}$ for example, can cross cell membranes through aquaporin channels and gap-junctions and diffuse freely between the cells $\mathbf{s}^{14,15}$. 
Similar to what happens in other physiological processes such as growth, inflammation and ageing, wound-induced ROS, and more specifically $\mathrm{H}_{2} \mathrm{O}_{2}$ with a rather long half-life, can play a regulating role in early wound responses and regeneration. In 2013, Love et al. demonstrated that amputation-induced ROS production is absolutely required for Xenopus tadpole tail regeneration ${ }^{16}$. Shortly after that, Gauron and colleagues showed a similar ROS production at the amputation site during fin regeneration in adult zebrafish and proved its role in blastema formation ${ }^{17}$. More recently, the Serras group demonstrated the presence of an oxidative burst just minutes after inducing regeneration of the wing imaginal disc in Drosophila ${ }^{18}$. Also in planarians, we observed that an amputation-induced ROS burst is necessary for proper stem cell differentiation and successful regeneration ${ }^{13}$.

Many studies already indicated an interplay between ROS and mitogenactivated protein kinases (MAPK) signaling pathways ${ }^{18-22}$. Ruffels et al. simulated a ROS burst by direct exposure of exogenous $\mathrm{H}_{2} \mathrm{O}_{2}$ to human neuroblastoma cells, resulting in a significant increase of ERK activation. In Drosophila, ROS-dependent stimulation of MAPKs is essential for the activation of JAK/STAT signaling, which drives regeneration. In addition, literature shows that the activation of MAPK pathways can be mediated by the upstream epidermal growth factor receptor (EGFR) in planarians ${ }^{24,25}$ and other models ${ }^{26-29}$. Also, the activation of the EGFR pathway by ROS has been described in different models ${ }^{30,31}$. Furthermore, previous studies have identified a functional relationship between the EGFR pathway and the egr (early growth response) family of transcription factors in planarian regeneration, in which a link between egr genes and ERK activity has been demonstrated in different models ${ }^{32-35}$. Overall, these reports suggest that wound-induced ROS signaling operates through EGFR-MAPK pathways in order to stimulate transcriptional expression of cytokines that in turn will be crucial to trigger tissue repair and restore homeostasis ${ }^{19}$.

Even though ROS, EGFR and ERK have been shown to be required for planarian regeneration, it is not known the relationship between these pathways. Here, we show that amputation-induced ROS production might be the upstream cue that would activate ERK signaling to initiate regeneration in these animals. 


\section{Results}

\section{Generation of Reactive Oxygen Species after applying an R - or H wound}

Recently, it was shown that a common generic wound response program is triggered by both injuries that require only wound healing ( $\mathrm{H}$-wounds) and by injuries that imply tissue loss and, therefore, require regeneration (R-wounds) ${ }^{10}$. In previous research, we showed an amputation-induced ROS burst after inflicting an R-wound in planarians ${ }^{13}$. Here, we confirm the fast ROS production at the R-wound site (Fig. 1A.1), and additionally show that a ROS burst occurs after applying an H-wound (Fig. 1A.2). Negative controls (without carboxy-H2DCFDA), showed no autofluorescence at both $\mathrm{R}$ - and $\mathrm{H}$-wound sites (Suppl. Fig. 1).

Owlarn and colleagues have demonstrated the triggering of generic initiationsignals in both wound types in dormant, MEK-inhibited fragments ${ }^{10}$. Therefore, it is important to verify the presence of ROS at the wound site after rewounding MEKinhibited fragments in order to analyze its possible role in the rescue of regeneration. ROS were indeed detected at the site of the newly inflicted R-wound (Fig. 1B.1, 16/16) as well as H-wound (Fig. 1B.2, 16/16) in these MEK-inhibited fragments. Interestingly, ROS levels were not only increased at the wound site after inflicting an $\mathrm{H}$-wound, (Fig. 1B.3, red square) but also occurred at the original, dormant, R-wound sites (Fig.1B.3, red arrow head, 11/16). To confirm the ROS signal, DPI was used as a negative control (Fig. 1C, 13/13).

In summary, these results, together with the observed regenerative impairments after ROS inhibition (Suppl. Fig. 2) ${ }^{13}$, point out the fast developed, wound-induced ROS as putative upstream key mediator in both initiating regeneration in control animals and in the rescue of regeneration in dormant MEKinhibited fragments.

\section{Hydrogen peroxide treatment rescues regeneration in dormant MEK-inhibited}

\section{fragments}

To functionally confirm a role for ROS as a regeneration initiating signal, we investigated if we could reactivate MEK-inhibited, dormant blastemas by treating them with an exogenous ROS-source. The in vivo ROS stain that we addressed to visualise wound-induced ROS production detects a broad range of reactive oxygen species, making it difficult to specify which ROS to use. However, because of the beneficial characteristics of $\mathrm{H}_{2} \mathrm{O}_{2}$ for its function as messenger molecule and the 
supporting evidence regarding the activation potency for several molecular pathways, we focused on using $\mathrm{H}_{2} \mathrm{O}_{2}$ as exogenous ROS-source (Fig. 2).

When inflicting a new R-wound (Fig. 2B: REcut) to MEK-inhibited trunk fragments (with pharynx), 96,88\% $(n=32)$ of the fragments regenerated. A small fraction of these animals $(6,25 \%)$ started regenerating, but neither eyes nor pharynx could be identified at the moment of comparison. In tail fragments (without a pharynx), $100 \%(n=30)$ restarted regeneration after inflicting an R-wound, of which $96,67 \%$ regenerated normally. In the absence of a new R-wound (Fig. 2B: $\mathrm{NO} \mathrm{H}_{2} \mathrm{O}_{2}$ ), $91,67 \%$ ( $n=60)$ of the MEK-inhibited trunks failed to regenerate, while the remaining part of the worms regenerated slower. The same trend was visible in the tail fragments: $95 \%(n=80)$ of the fragments did not regenerate and in $5 \%$ regeneration was delayed. In all cases, the absence of regeneration remained for at least 21 days. Initial dose-response experiments indicated that a 6 hours treatment with 1,5 to $2,25 \mathrm{mM} \mathrm{H}_{2} \mathrm{O}_{2}$ was enough to fully rescue $26,76 \%(n=120)$ of the trunk fragments (with a pharynx), and $5 \%(n=80)$ of tail fragments (without a pharynx). An additional $20,83 \%$ of the trunk fragments were partially rescued, showing a slower regeneration. In case of the tail fragments this partial rescue reached $28,75 \%$. In both setups, trunk and tail fragments, a small amount of animals died; $6,58 \%$ and $13,75 \%$ respectively. In order to exclude wounding by $\mathrm{H}_{2} \mathrm{O}_{2}$, a ROS staining was performed on dormant fragments after 3 and 6 hours of exposure to $\mathrm{H}_{2} \mathrm{O}_{2}$. No ROSinduced wounding was observed at the epidermis of the $\mathrm{H}_{2} \mathrm{O}_{2}$-treated planarians (Suppl. Fig. 3).

Taken together, these results show the potency of $\mathrm{H}_{2} \mathrm{O}_{2}$ in reversing MEKinhibition, consequently rescuing regeneration. Hereby, it supports the finding of ROS as upstream regeneration-initiation signal.

\section{Inhibition of ROS production blocks ERK activation at the wound site}

To identify downstream targets and a possible interaction between the amputationinduced ROS burst and ERK, we performed an immunostaining with an anti-pERK antibody after ROS inhibition with $3 \mu \mathrm{M} \mathrm{DPI}^{23} 24$. In the control conditions, where animals were kept in regular medium or DMSO, an activation of $\mathrm{pERK}$ was observed at the wound site of head- (control: 4/5, DMSO: 3/6), trunk- (control: 6/7, DMSO: 6/8) as well as tail pieces (control: 4/6, DMSO: 4/6). This pERK-sinal was strongly reduced in the DPI-treated fragments (heads: 5/7, trunks: $7 / 9$, tails: $5 / 7$ ) (Fig. 3B), 
suggesting that an amputation-induced ROS production at the wound site is required for the phosphorylation and proper activation of ERK (Fig. 3C).

\section{Smed-egfr-3 and Smed-egr-4 silencing impair ROS production and pERK activation in regenerating animals}

Literature has already described examples of the activation of EGFR signaling by ROS and the potential of EGFR in mediating MAPK signaling including ERK activation $^{24-31}$. Additionally, the egr (early growth response) family of transcription factors is suggested to be a downstream target of EGFR signaling in planarian regeneration as well as in other models ${ }^{32-35}$.

In order to further investigate the functional relationship of Smed-egfr-3 and Smed-egr-4 with pERK, we carried out an immunostaining with the anti-pERK antibody in controls and RNAi-mediated Smed-egfr-3 and Smed-egr-4 knockdown animals. (Fig. 4A). A clear pERK activation was observed in all control fragments at 6HPA (trunk pieces: 7/11, tail pieces: 7/8) and 1DPA (trunk pieces:8/9, tail pieces: $11 / 14)$. In contrast, a strong reduction of the anti-pERK signal at the wound site was observed in Smed-egfr-3 silenced fragments at 6HPA (trunk pieces: 5/5, tail pieces: 5/7) and 1DPA (trunk pieces:7/8, tail pieces:12/14). Similar results were observed after silencing Smed-egr-4, both at 6HPA (trunk pieces: 9/9, tail pieces: $7 / 7$ ) and 1DPA (trunk pieces:6/7, tail pieces:8/9) (Fig. 4B). These results suggest that Smedegfr-3 and Smed-egr-4 are required for ERK activation (Fig. 4C).

To characterize the link of ROS with EGFR signaling during planarian regeneration, an In vivo ROS visualisation was performed in controls and animals subjected to RNAi silencing of either Smed-egfr-3 or Smed-egr-4. (Fig. 5). Whereas the presence of the amputation-induced ROS burst was clear in the control animals (30MPA: 5/7, 6HPA: 7/8, 24HPA: 6/8), trunk fragments subjected to Smed-egfr-3 knockdown showed a clearly diminished ROS production at all time points (30MPA: 6/7, 6HPA: 5/7, 24HPA: 5/7). Similar results were observed in Smed-egr-4 RNAi animals (30MPA: 6/9, 6HPA: 4/7, 24HPA: 5/8) (Fig. 5B).

Altogether, these results indicate that Smed-egfr-3 as well as Smed-egr-4 might play a pivotal role in regulating the amputation-induced ROS production, possibly through the existence of feedback mechanisms (Fig. 5C). Furthermore, it is suggested that the activation of pERK by ROS could be mediated by the EGFR pathway during regeneration. Additionally, results point out the existence of a 
second, negative feedback mechanism of egr- 4 in order to regulate pERK activation as well (Fig. 4C).

\section{MAPK phosphatase is overexpressed in Smed-egr-4 knockdown animals}

In the context of previously suggested negative feedback mechanism of egr-4 in relation to ERKs activation, a study of Tasaki et al. 2011 showed that in planarians pERK could be negatively regulated by the action of a MAPK phosphatase (MKP) $)^{23}$. Therefore, the expression of Smed-mkp was checked in controls and planarians subjected to knockdown of either Smed-egfr-3 or Smed-egr-4. A strongly increase of Smed-mkp expression was observed at the anterior wound site of the regenerating trunk fragments in Smed-egr-4 RNAi animals $(n=4 / 4)$ in comparison to no expression in both control $(n=5 / 5)$ and Smed-egfr-3 RNAi $(n=3 / 3)$ animals (Fig. 5D). These results suggest that egr-4 might be required to maintain $\mathrm{pERK}$ activation by inhibiting Smed-mkp.

\section{Discussion}

The complex process of animal regeneration is highly organized and regulated by a tightly controlled network of signaling pathways, of which we have only discovered the tip of the iceberg. Till now, regeneration research has mainly focused on molecular mechanisms regulating cell fate, polarity re-establishment, tissue differentiation and organ positioning, while the question on what initially triggers regeneration remains unanswered in many cases. In recent years several studies have reported the indispensability of reactive oxygen species (ROS) during regeneration. Amputation-induced ROS production was observed in early tail regeneration of Xenopus tadpoles and in fin regeneration of adult zebrafish ${ }^{16,17}$. Similar observations were reported during the regeneration process of the wing imaginal disc in Drosophila and during anterior and posterior regeneration of the planarian Schmidtea mediterranea ${ }^{13,18}$. In all of the above-mentioned cases, ROS production was clearly linked to the capacity to regenerate, e.g. the absence of ROS production during regeneration-initiation led to regenerative impairments (Suppl. Fig. 1) $10,13,16-18$. 
Previously we showed a rapid ROS burst at the wound site after inflicting a regenerative(R)-wound as well as its necessity for proper regeneration in planarians $^{13}$. Here, we demonstrate that ROS are also produced after healing $(\mathrm{H})$ wounding (Fig. 1A). Owlarn and colleagues showed that in planarians both Rwounds and $\mathrm{H}$-wounds trigger a common initial molecular response mediated by ERK signaling. Inhibition of the ERK upstream activator, MAPK/ERK kinase (MEK), completely blocks regeneration after an R-wound ${ }^{10}$. No blastema is initiated and the body fragments remain "dormant" until a new R-wound or H-wound is made. The fact that inflicting a new $\mathrm{H}$-wound is capable of rescuing regeneration of dormant fragments suggests that, in planarians, any kind of wounding (whether or not it results in tissue loss) triggers an early response through ERK activation and only in the context of tissue loss this is followed by a late regenerative response that leads to the restoration of the missing part(s) ${ }^{10}$. However, the initial upstream signal that activates ERK, remained unknown. Here, we show a ROS burst in the MEK-inhibited dormant fragments after inflicting a new $\mathrm{H}$ - or R-wound (Fig. 1B). Surprisingly, Hwounding not only induces a ROS burst at the $\mathrm{H}$-wound site but also at the original, dormant R-wound site (Fig. 1B3), outlining again the involvement of ROS in the initiation of regeneration. However, in planarians it is not yet clear if ROS can propagate as signaling molecules by themselves or if they induce an unknown secondary signal to the dormant wound site in order to locally restart regeneration $^{14,15,19}$.

Generally it is stated that ROS function as messenger molecules and regulate processes such as cell proliferation, differentiation and patterning by modulating gene transcription and protein phosphorylation ${ }^{11,12,19,36-39}$. In many of these pathways, MAPKs are predominantly mentioned as a required intermediate link, playing pivotal roles in signal transduction from the cell membrane to the nucleus. Exogenous addition of $\mathrm{H}_{2} \mathrm{O}_{2}$ or treatment with ROS-inducing compounds, lead to the activation of the MAPK pathway ${ }^{19,40,41}$. On the other hand, the inhibition of ROS production or the stimulation of antioxidant defense mechanisms, block MAPK activation $^{20,42}$. Taking into account that, in planarians, ROS are necessary for regeneration, that ROS are induced very early after $\mathrm{H}$ - and R-wounding, and that 
ERK activation is required for regeneration, we searched to determine whether ROS act upstream of ERK and which factors mediate ROS production and ERK activation. Our results show that MEK-inhibited dormant tails can be rescued by the addition of exogenous $\mathrm{H}_{2} \mathrm{O}_{2}$ without the need to inflict a new wound (Fig. 2). It is important to point out that the $\mathrm{H}_{2} \mathrm{O}_{2}$ treatment did not induce wounds in the epidermis, indicating that the rescue was not introduced by re-wounding the dormant tails (Suppl. Fig.3). We hypothesize that because of its relatively long half-life and good membrane permeability, $\mathrm{H}_{2} \mathrm{O}_{2}$ functions as secondary messenger and triggers the activation of important regeneration-related downstream signaling processes such as ERK activation. The strong reduction of ERK activation at the wound site after inhibition of ROS production in regenerating animals, again verifies the upstream function of ROS relative to ERK (Fig. 3).

In many organisms, the epidermal growth factor receptors (EGFR), one of families of the receptor tyrosine kinases (RTK), are known to regulate several biological processes by activating key downstream pathways including the MAPK pathway ${ }^{26-29,43-45}$. In planarians, Smed-egfr-3 is required for proper regeneration as well as for ERK activation (Fig.4) $)^{24,46}$. Recent literature suggested that RTKassociated activation mechanisms are under redox control. Activation of EGFR signaling by ROS can occur in at several ways ${ }^{47}$. On one hand, intracellular ROS can facilitate the phosphorylation of EGFR and induce a subsequent cascade of phosphorylations. According to Peus and colleagues, $\mathrm{H}_{2} \mathrm{O}_{2}$ specifically acts as a critical mediator in this case ${ }^{48}$. The absence of ERK activation after inhibition of ROS production (Fig.3), and the rescue of regeneration in dormant tails by $\mathrm{H}_{2} \mathrm{O}_{2}$ in our results support this hypothesis (Fig.2). Furthermore, silencing Smed-egfr-3 results in decreased amputation-induced ROS production, suggesting the dependency of ROS production on the EGFR signaling (Fig.5). This could be explained by the fact that ligand-dependent dimerization of EGFR induces ROS production for its autophosphorylation and consequent activation, leading to a decreased ROS production when silenced ${ }^{47}$. However, literature also suggests that $\mathrm{H}_{2} \mathrm{O}_{2}$ acts as a critical mediator in the ligand-independent phosphorylation and activation of EGFR ${ }^{48}$. Additionally, EGFR signaling can also be redox-controlled via MAPK phosphatases 
(MKPs), responsible for the dephosphorylation and inactivation of ERK. MPKs contain catalytic cysteine residues which are targets for oxidation by ROS leading to its deactivation ${ }^{19,49}$. As a result, ROS inhibition would lead to increased MKP activity and therefore inhibition of ERK activation.

Our results suggest a second regulator of MKP activity, namely Smed-egr-4. Smed-egr-4, codes for a zinc finger transcription factor of the early growth response factor family and is reported as a putative target of Smed-egfr- $3^{32}$. The transcription factor is upregulated immediately after inflicting an $\mathrm{R}$ - or $\mathrm{H}$-wound, while hardly expressed in intact planarians ${ }^{50,51}$. Smed-egr-4 silencing results in regeneration defects similar to those after Smed-egfr-3 downregulation, inhibition of ERKs activation and decreased ROS production ${ }^{13,23,32,46}$. Silencing of Smed-egr-4 leads to a strong reduction of ERK activation together with a pronounced upregulation of Smed-mkp (Fig. 5) which suggests that Smed-egr-4 might function as an inhibitor of MKP activity, preventing the dephosphorylation and inactivation of ERK. Together with ROS they form a secure and self-reinforcing regulation mechanism of MKP activation state. Moreover, the silencing of Smed-egr-4 also leads to a reduced amputation-induced ROS production which suggest the existence of a feedback mechanism regulating ROS production by Smed-egr-4 (Fig. 4). Because regulation of NADPH-oxidases (Nox) expression can be controlled by ERK-activation and linked transcription factors, Smed-egr-4 knockdown which leads to increased MKP activation and consequently decreased ERK activation, can alter Nox-expression, explaining the impaired ROS production ${ }^{53}$. However, further experiments are necessary to clarify the involvement of Nox genes in planarians. All these data together with what is known about ROS and EGFR-MAPK signaling in other systems allows us to propose a model for the interactions of all these elements during planarian regeneration (Fig. 6).

In summary, our results suggest that: i) ROS have the potential to rescue regeneration in MEK-inhibited dormant tails, ii) ROS are required for ERK activation at early regeneration stages, iii) the EGFR pathway can mediate ROS production with ERK activation during planarian regeneration. We provide the first evidence of amputation- and wound-induced ROS production in relationship with the EGFR- 
MAPK signaling pathway during planarian regeneration in which ROS not only are identified as most upstream trigger for regeneration-initiation, but additionally also perform its functions more downstream.

\section{Materials and Methods}

\section{Planarian cultivation}

An asexual strain of the freshwater planarian species Schmidtea mediterranea was kept in Milli-Q water containing $1.2 \mathrm{mM} \mathrm{NaHCO}_{3}, 1.6 \mathrm{mM} \mathrm{NaCl}, 1.0 \mathrm{mM} \mathrm{CaCl}_{2}, 1.0$ $\mathrm{mM} \mathrm{MgSO}_{4}, 0.1 \mathrm{mM} \mathrm{MgCl}_{2}$ and $0.1 \mathrm{mM} \mathrm{KCl}$ (cultivation medium). The planarians were continuously maintained in the dark at a temperature of $20^{\circ} \mathrm{C}$. Once a week they were fed with veal liver. Animals used in experiments were starved for at least 7 days before the procedure.

\section{Inhibition of Reactive Oxygen Species (ROS) Production}

The nonspecific flavoprotein inhibitor, Diphenyleneiodonium chloride (DPI, Sigma Aldrich, D2926), was used in order to block ROS production by interfering with several electron transporters. Animals were exposed to $3 \mu \mathrm{M}$ DPI for 5 hours prior to in vivo ROS staining and 1hour prior amputation when followed by $\mathrm{pERK}$ immunohistochemistry. In both cases, animals were continuously exposed to DPI during the whole regeneration period. Because of its hydrophobic character, DPI was prepared in 0.01\% dimethylsulfoxide (DMSO, Sigma Aldrich, 471267). In all experiments concerning DPI exposure, a DMSO-exposed control group was added to take into account the possible effects of DMSO since relatively high concentrations can have neurotoxic effects and influence cell proliferation in $S$. mediterranea ${ }^{52}$.

\section{Immunohistochemistry}

To analyse the relationships between ROS, Smed-egfr-3 and Smed-egr-4 relative to ERK activation in early regeneration, a pERK immunostaining was performed after interfering with the aforesaid. Planarians were amputated followed by 6 and/or 24 hours of regeneration. Next, they were fixed and processed as previously described by Fraguas et al. ${ }^{24}$. Bleached animals were washed with PBSTx (1x PBS (10x PBS: $1,37 \mathrm{M} \mathrm{NaCl}, 27 \mathrm{mM} \mathrm{KCl}, 100 \mathrm{mM} \mathrm{Na}_{2} \mathrm{HPO}_{4}, 20 \mathrm{mM} \mathrm{KH}_{2} \mathrm{PO}_{4}$ in ultrapure $\mathrm{H}_{2} \mathrm{O}$ ) and 
incubated for 4 hours in $1 \%$ blocking solution (1\% BSA in PBSTx) followed by the primary antibody (anti-pERK23 diluted 1/1000 in blocking solution) overnight at $4^{\circ} \mathrm{C}$. After PBSTx washes and 1 hour in blocking solution, they were incubated with the secondary antibody (goat anti-rabbit-POD diluted $1 / 500$ in blocking solution) overnight at $4^{\circ} \mathrm{C}$. After PBSTx washes, samples were incubated for 8 minutes in TSA Plus Fluorescein solution (1/50 TSA Plus Fluorescein in 1x Amplification Buffer (Tyramide Signal Amplification Labeling Kit No. 2; Molecular Probes, Thermo Fisher Scientific)) in darkness. Samples were mounted after the final PBSTx washes (RT) and analysed with a MZ16F fluorescence stereomicroscope (Leica) equipped with a ProgRes C3 camera (Jenoptik, Jena, Germany).

\section{MEK Inhibition}

The chemical compound PD0325901 (Calbiochem) was used to reversibly inhibit MEK activity and subsequently prevent the phosphorylation and activation of ERK. As a consequence we obtained dormant planarian fragments as described by Owlarn et al. ${ }^{10}$. PD0325901 was dissolved in DMSO and used in a concentration of $25 \mu \mathrm{M}$. Planarians were exposed to PD0325901 for 1 hour prior and up to 5 to 7 days post amputation. The exposure solution was replaced daily or every two days, depending on the experiment. After treatment with PD0325901, animals were gently washed and placed into fresh medium until used for experiments.

\section{$\mathrm{H}_{2} \mathrm{O}_{2}$ Treatment}

Dormant, MEK-inhibited fragments were exposed to $\mathrm{H}_{2} \mathrm{O}_{2}$ with the intention to rescue regeneration. After initial range finding experiments, dormant fragments were exposed to either $1,5 \mathrm{mM}(0,005 \%)$ or $2,25 \mathrm{mM}(0,0075 \%) \mathrm{H}_{2} \mathrm{O}_{2}$ (in cultivation medium) for 6 hours. After 3 washes with cultivation medium, they were kept in fresh medium.

\section{Reactive Oxygen Species (ROS) Detection}

The compound 5-(and-6)-carboxy-2',7'-dichlorodihydrofluorescein diacetate (carboxy- $\mathrm{H}_{2}$ DCFDA, Image-iT LIVE Green Reactive Oxygen Species Detection Kit, Molecular Probes; Invitrogen, 136007) was used to visualise the in vivo production of ROS, in which fluorescent carboxy-DCF is produced through ROS oxidation. The ROS visualisation procedure was performed on Smed-egfr-3 and Smed-egr-4 RNAi knockdown animals as well as control - and MEK-inhibited animals either combined 
or not with the inhibition of ROS production by DPI. Animals were exposed to carboxy- $\mathrm{H}_{2}$ DCFDA $(25 \mu \mathrm{M}, 1 \mathrm{ml})$ for 1 hour prior to amputation and for 1 day post RNAi. Amputated animals were again incubated in carboxy- $\mathrm{H}_{2}$ DCFDA for 15 minutes before immobilisation in 2\% low melting point (LMP) agarose (Invitrogen, 16520050). Imaging of the samples was performed on 30 minutes, 6 - and/or 24 hours post amputation (MPA/HPA) using a MZ16F fluorescence stereomicroscope (Leica) combined with a ProgRes C3 camera (Jenoptik, Jena, Germany) or a Ts2-FL inverted microscope (Nikon) combined with a Ds-Fi3 colour camera (Nikon). For all pictures the exact same capturing settings were used. Additionally, all experiments were also performed without the carboxy-H2DCFDA-stain in order to discard possible autofluorescence at the wound sites.

\section{RNA Interference}

Double-stranded RNA (dsRNA) for Smed-egfr-3 and Smed-egr-4 were synthesised as previously described (Smed-egfr-3, forward primer: GTACTGGGCAATGTTGGACCTGGC, primer: TGACGGCCTCATGTGGGGATCATCG; smed-egr-4, forward primer: GGCCGCGGTATGGGATATTCTTCTCAACTG: primer: GTAATTATGAGTCGTGTAGGC). Animals were injected in two rounds of 3 consecutive days each with 4 days elapsed in between. On day 4 of the second round, planarians were amputated pre- and post-pharyngeally to induce regeneration. Injections were done using the Nanoject II (Drummond Scientific, Broomall, PA, USA) and consisted of three times $32 \mathrm{nl}$ containing $1 \mu \mathrm{g} / \mu \mathrm{l}$ dsRNA. Controls were injected with dsRNA of $g f p$.

\section{In Situ Hybridization}

Riboprobes for in situ hybridizations were synthesized using the DIG RNA labelling kit (Sp6/T7, Roche) following the manufacturer's instructions. Primers used for the development of the DIG-labeled Smed-MKP probe were the following; forward primer: GACAATTTACGTTGTCCAACA, primer: GTCCGGCGCCGTTTGACCCA.

To perform whole mount in situ hybridizations, regenerated trunk fragments were fixed at 6 hours post amputation (6HPA), using ice cold $2 \% \mathrm{HCl}$ (ultrapure $\mathrm{H}_{2} \mathrm{O}, 5$ minutes). Next, they were put in Carnoy's solution (60\% ethanol, $10 \%$ acetic acid 
and $30 \%$ chloroform) for 2 hours on $4^{\circ} \mathrm{C}$. After 1 hour incubation in $100 \% \mathrm{MeOH}$ at $20{ }^{\circ} \mathrm{C}$, samples were washed and bleached in $6 \% \mathrm{H}_{2} \mathrm{O}_{2}(\mathrm{MeOH})$ for $16 \mathrm{~h}$. Animals were rehydrated through a series of ethanol washes followed by PBSTween $(10 \%)$ whashes. Afterwards, animals were treated with $20 \mu \mathrm{g} / \mathrm{ml}$ proteinase $\mathrm{K}$ (Ambion)/PBSTween for 10 minutes at RT. The proteinase K/PBSTween was removed with a PBSTween $\left(5\right.$ minutes, $\left.4^{\circ} \mathrm{C}\right)$ washing step and postfixed in $4 \%$ paraformaldehyde (PFA)/PBS for one hour at $4^{\circ} \mathrm{C}$. Tissues were acetylated by 20 minutes of incubation in $0.1 \mathrm{mM}$ TEA after which $25 \mu \mathrm{l}$ of acetic anhydride was added. After 10 additional minutes another $25 \mu$ of acetic anhydride was added. All the steps including TEA were performed at room temperature. Animals were washed with PBSTween (10 minutes, RT). Next, PBSTween was replaced by $50 \%$ prehybridisation buffer (in PBSTween) followed by incubation in $100 \%$ prehybridisation buffer ( $50 \%$ formamide, $5 \times$ SSC, $0.1 \mathrm{mg} / \mathrm{ml}$ Yeast tRNA, $0.1 \mathrm{mg} / \mathrm{ml}$ heparina, $0.1 \%$ Tween 20, $10 \mathrm{mM}$ DTT) for 2 hours at $56{ }^{\circ} \mathrm{C}$. Hybridization was carried out for 16 hours at $56{ }^{\circ} \mathrm{C}$ in hybridization buffer (prehybridisation buffer + 10\% Dextran Sulphate and the DIG-labelled probe). Afterwards, samples were washed through a series of posthybridisation buffers and buffer I ( $0.1 \%$ Triton $\times 100$, Maleic acid $11.6 \mathrm{~g}, \mathrm{NaCl} 9.76 \mathrm{~g}, 2 \mathrm{~N} \mathrm{NaOH} 95 \mathrm{ml}$ )/1000ml) and blocked during a $1 \mathrm{~h}$ incubation in Buffer II (Buffer I with 10\% Blocking Solution). Samples were incubated at RT for 3 hours in 1:2000 anti-DIG/Buffer II. Colour development was performed by incubation of the samples in $20 \mu \mathrm{IBT} / \mathrm{BCIP}$ stock solution/ml TMN at RT. When the colour reaction reached the desired state, the animals were washed with PBS and fixed in 4\% PFA/PBS. To optimize the colour development, a series of ethanol washes was performed. Finally, the samples were washed with PBS and kept in $70 \%$ glycerol/PBS at $4^{\circ} \mathrm{C}$. Samples were analysed and bright-field images were digitized using the MZ16F stereomicroscope (Leica) equipped with a ProgRes C3 camera (Jenoptik, Jena, Germany).

\section{Data availability}

The authors declare that all data supporting the findings of this study are available within the article and its Supplementary Information files or from the corresponding author upon reasonable request. 


\section{Acknowledgements}

The authors thank Yoshihiko Umesono for the anti-pERK antibody and Natascha Steffanie and Ria Vanderspikken for their skillful technical assistance. This work was financially supported by grants BFU2015-65704P and PGC2018-100747-B-100 to F.C. (Ministerio de Ciencia, Innovación y Universidades, Spain), and by FWO $(1522015 \mathrm{~N}, 1522719 \mathrm{~N}$ and GOB8317N) and BOF UHasselt to K.S. The research leading to results presented in this publication was carried out with infrastructure funded by EMBRC Belgium - FWO project GOH3817N to K.S.

\section{References}

1. Morgan TH. Regeneration and Liability to Injury. Science. 1901;14(346):23548.

2. Levin M. Large-scale biophysics: ion flows and regeneration. Trends in cell biology. 2007;17(6):261-70.

3. Birnbaum KD, Sanchez Alvarado A. Slicing across kingdoms: regeneration in plants and animals. Cell. 2008;132(4):697-710.

4. Galliot B. Injury-induced asymmetric cell death as a driving force for head regeneration in Hydra. Dev Genes Evol 2013; 223(1-2): 39-52.

5. Endo T, Bryant SV, Gardiner DM. A stepwise model system for limb regeneration. Dev Biol 2004; 270(1): 135-145.

6. Brockes JP, Kumar A. Comparative aspects of animal regeneration. Annu Rev Cell Dev Biol 2008; 24: 525-549.

7. Gurley KA, Rink JC, Sanchez Alvarado A. Beta-catenin defines head versus tail identity during planarian regeneration and homeostasis. Science. 2008;319(5861):323-7.

8. Rink JC, Gurley KA, Elliott SA, Sanchez Alvarado A. Planarian Hh signaling regulates regeneration polarity and links $\mathrm{Hh}$ pathway evolution to cilia. Science. 2009;326(5958):1406-10.

9. Reddien PW, Bermange AL, Kicza AM, Sanchez Alvarado A. BMP signaling regulates the dorsal planarian midline and is needed for asymmetric regeneration. Development. 2007;134(22):4043-51. 
10. Owlarn S, Klenner F, Schmidt D, Rabert F, Tomasso A, Reuter H, et al. Generic wound signals initiate regeneration in missing-tissue contexts. Nat Commun. 2017;8(1):2282.

11. Bedard K, Krause KH. The NOX family of ROS-generating NADPH oxidases: physiology and pathophysiology. Physiol Rev. 2007;87(1):245-313.

12. Kennedy KA, Sandiford SD, Skerjanc IS, Li SS. Reactive oxygen species and the neuronal fate. Cellular and molecular life sciences : CMLS. 2012;69(2):215-21.

13. Pirotte N, Stevens AS, Fraguas S, Plusquin M, Van Roten A, Van Belleghem $F$, et al. Reactive Oxygen Species in Planarian Regeneration: An Upstream Necessity for Correct Patterning and Brain Formation. Oxid Med Cell Longev. 2015;2015:392476.

14. Tamma G, Valenti G, Grossini E, Donnini S, Marino A, Marinelli RA, et al. Aquaporin Membrane Channels in Oxidative Stress, Cell Signaling, and Aging: Recent Advances and Research Trends. Oxid Med Cell Longev. 2018;2018:1501847.

15. Bienert GP, Moller AL, Kristiansen KA, Schulz A, Moller IM, Schjoerring JK, et al. Specific aquaporins facilitate the diffusion of hydrogen peroxide across membranes. J Biol Chem. 2007;282(2):1183-92.

16. Love NR, Chen Y, Ishibashi S, Kritsiligkou P, Lea R, Koh Y, et al. Amputationinduced reactive oxygen species are required for successful Xenopus tadpole tail regeneration. Nat Cell Biol. 2013;15(2):222-8.

17. Gauron C, Rampon C, Bouzaffour M, Ipendey E, Teillon J, Volovitch M, et al. Sustained production of ROS triggers compensatory proliferation and is required for regeneration to proceed. Sci Rep. 2013;3:2084.

18. Santabarbara-Ruiz P, Lopez-Santillan M, Martinez-Rodriguez I, BinaguiCasas A, Perez L, Milan M, et al. ROS-Induced JNK and p38 Signaling Is Required for Unpaired Cytokine Activation during Drosophila Regeneration. PLoS Genet. 2015;11(10):e1005595.

19. Torres M, Forman HJ. Redox signaling and the MAP kinase pathways. Biofactors. 2003;17(1-4):287-96.

20.Son Y, Cheong YK, Kim NH, Chung HT, Kang DG, Pae HO. MitogenActivated Protein Kinases and Reactive Oxygen Species: How Can ROS Activate MAPK Pathways? J Signal Transduct. 2011;2011:792639. 
21. Ito K, Hirao A, Arai F, Matsuoka S, Takubo K, Hamaguchi I, et al. Regulation of oxidative stress by ATM is required for self-renewal of haematopoietic stem cells. Nature. 2004;431(7011):997-1002.

22. Ruffels J, Griffin M, Dickenson JM. Activation of ERK1/2, JNK and PKB by hydrogen peroxide in human SH-SY5Y neuroblastoma cells: role of ERK1/2 in H2O2-induced cell death. Eur J Pharmacol. 2004;483(2-3):163-73.

23. Tasaki J, Shibata N, Nishimura O, Itomi K, Tabata Y, Son F, et al. ERK signaling controls blastema cell differentiation during planarian regeneration. Development. 2011;138(12):2417-27.

24. Fraguas S, Umesono Y, Agata K, Cebria F. Analyzing pERK Activation During Planarian Regeneration. Methods Mol Biol. 2017;1487:303-15.

25. Barberan S, Fraguas S, Cebria F. The EGFR signaling pathway controls gut progenitor differentiation during planarian regeneration and homeostasis. Development. 2016;143(12):2089-102.

26. Jin Y, Ha N, Fores M, Xiang J, Glasser C, Maldera J, et al. EGFR/Ras Signaling Controls Drosophila Intestinal Stem Cell Proliferation via CapicuaRegulated Genes. PLoS Genet. 2015;11(12):e1005634.

27. Geiger JA, Carvalho L, Campos I, Santos AC, Jacinto A. Hole-in-one mutant phenotypes link EGFR/ERK signaling to epithelial tissue repair in Drosophila. PloS one. 2011;6(11):e28349.

28. Underwood RS, Deng Y, Greenwald I. Integration of EGFR and LIN-12/Notch Signaling by LIN-1/Elk1, the Cdk8 Kinase Module, and SUR-2/Med23 in Vulval Precursor Cell Fate Patterning in Caenorhabditis elegans. Genetics. 2017;207(4):1473-88.

29. Patel AL, Shvartsman SY. Outstanding questions in developmental ERK signaling. Development. 2018;145(14).

30. Aslan M, Ozben T. Oxidants in receptor tyrosine kinase signal transduction pathways. Antioxid Redox Signal. 2003;5(6):781-8.

31. Nakashima I, Takeda K, Kawamoto Y, Okuno Y, Kato M, Suzuki H. Redox control of catalytic activities of membrane-associated protein tyrosine kinases. Arch Biochem Biophys. 2005;434(1):3-10.

32. Fraguas S, Barberan S, Iglesias M, Rodriguez-Esteban G, Cebria F. egr-4, a target of EGFR signaling, is required for the formation of the brain primordia and head regeneration in planarians. Development. 2014;141(9):1835-47. 
33. Corson LB, Yamanaka Y, Lai KM, Rossant J. Spatial and temporal patterns of ERK signaling during mouse embryogenesis. Development. 2003;130(19):4527-37.

34. Dussmann P, Pagel JI, Vogel S, Magnusson T, Zimmermann R, Wagner E, et al. Live in vivo imaging of Egr-1 promoter activity during neonatal development, liver regeneration and wound healing. BMC Dev Biol. 2011;11:28.

35. Wung BS, Cheng JJ, Chao YJ, Hsieh HJ, Wang DL. Modulation of Ras/Raf/extracellular signal-regulated kinase pathway by reactive oxygen species is involved in cyclic strain-induced early growth response-1 gene expression in endothelial cells. Circ Res. 1999;84(7):804-12.

36. Davis RJ. The mitogen-activated protein kinase signal transduction pathway. J Biol Chem. 1993;268(20):14553-6.

37. Hernandez-Garcia D, Castro-Obregon S, Gomez-Lopez S, Valencia C, Covarrubias L. Cell death activation during cavitation of embryoid bodies is mediated by hydrogen peroxide. Exp Cell Res. 2008;314(10):2090-9.

38. Boutros T, Chevet E, Metrakos P. Mitogen-activated protein (MAP) kinase/MAP kinase phosphatase regulation: roles in cell growth, death, and cancer. Pharmacol Rev. 2008;60(3):261-310.

39. Brown MD, Sacks DB. Protein scaffolds in MAP kinase signalling. Cell Signal. 2009;21(4):462-9.

40. Guyton KZ, Liu Y, Gorospe M, Xu Q, Holbrook NJ. Activation of mitogenactivated protein kinase by $\mathrm{H} 2 \mathrm{O} 2$. Role in cell survival following oxidant injury. J Biol Chem. 1996;271(8):4138-42.

41. Kamata $\mathrm{H}$, Hirata $\mathrm{H}$. Redox regulation of cellular signalling. Cell Signal. 1999;11(1):1-14.

42. Hashimoto S, Gon Y, Matsumoto K, Takeshita I, Horie T. N-acetylcysteine attenuates TNF-alpha-induced p38 MAP kinase activation and p38 MAP kinase-mediated IL-8 production by human pulmonary vascular endothelial cells. Br J Pharmacol. 2001;132(1):270-6.

43. Ramos JW. The regulation of extracellular signal-regulated kinase (ERK) in mammalian cells. Int J Biochem Cell Biol. 2008;40(12):2707-19. 
44. Osaki LH, Figueiredo PM, Alvares EP, Gama P. EGFR is involved in control of gastric cell proliferation through activation of MAPK and Src signalling pathways in early-weaned rats. Cell Prolif. 2011;44(2):174-82.

45. Walker F, Kato A, Gonez LJ, Hibbs ML, Pouliot N, Levitzki A, et al. Activation of the Ras/mitogen-activated protein kinase pathway by kinase-defective epidermal growth factor receptors results in cell survival but not proliferation. Mol Cell Biol. 1998;18(12):7192-204.

46. Fraguas S, Barberan S, Cebria F. EGFR signaling regulates cell proliferation, differentiation and morphogenesis during planarian regeneration and homeostasis. Dev Biol. 2011;354(1):87-101.

47. Heppner DE, van der Vliet A. Redox-dependent regulation of epidermal growth factor receptor signaling. Redox Biol. 2016;8:24-7.

48. Peus D, Meves A, Vasa RA, Beyerle A, O'Brien T, Pittelkow MR. H2O2 is required for UVB-induced EGF receptor and downstream signaling pathway activation. Free Radic Biol Med. 1999;27(11-12):1197-202.

49. Kamata H, Honda S, Maeda S, Chang L, Hirata H, Karin M. Reactive oxygen species promote TNFalpha-induced death and sustained JNK activation by inhibiting MAP kinase phosphatases. Cell. 2005;120(5):649-61.

50. Sandmann T, Vogg MC, Owlarn S, Boutros M, Bartscherer K. The headregeneration transcriptome of the planarian Schmidtea mediterranea. Genome Biol. 2011;12(8):R76.

51. Wenemoser D, Lapan SW, Wilkinson AW, Bell GW, Reddien PW. A molecular wound response program associated with regeneration initiation in planarians. Genes Dev. 2012;26(9):988-1002.

52. Stevens AS, Pirotte N, Plusquin M, Willems M, Neyens T, Artois T, et al. Toxicity profiles and solvent-toxicant interference in the planarian Schmidtea mediterranea after dimethylsulfoxide (DMSO) exposure. J Appl Toxicol. 2015;35(3):319-26.

53. Manea SA, Constantin A, Manda G, Sasson S, Manea A. Regulation of Nox enzymes expression in vascular pathophysiology: Focusing on transcription factors and epigenetic mechanisms. Redox Biol. 2015;5:358-66. 


\section{Figure legends}

\section{Figure 1}

In vivo visualisation of ROS production at the amputation site after rewounding MEK-inhibited fragments. For each condition, the experimental setup is displayed in the white boxes. Colour code as follows; green: MEK inhibition by PD0325901 (10 $\mu \mathrm{M}, 5$ days), red: inhibition of ROS production by DPI ( $3 \mu \mathrm{M}, 5$ hours), blue: ROS visualisation procedure using carboxy- $\mathrm{H}_{2}$ DCFDA, yellow: imaging procedure. The amputation setup is displayed in the dark grey boxes. Regenerative wound (R-wound) \& healing wound ( $\mathrm{H}$-wound). All animals were visualised 30 minutes post amputation (MPA). A representative close-up merged image of bright field and fluorescence of either R-wounds or $\mathrm{H}$-wounds is displayed on the right panel. (A) ROS production visualisation at the site of an R-wound (A.1) and an $\mathrm{H}$ wound (A.2) in controls. (B) ROS production visualisation at the amputation site of an R-wound (B.1) and an H-wound (B.2) in MEK-inhibited (PD0325901), "dormant" fragments. (B.3) Image of the site of the original R-wound applied before MEKinhibition (red arrowhead) together with the newly applied $\mathrm{H}$-wound (red square). (C) ROS production visualisation after rewounding (C.1: R-wound, C.2: H-wound) MEKinhibited and DPI exposed dormant fragments. Because of the strongly reduced fluorescence in (C), the close-up of the wound site is shown in bright field (upper panels) and fluorescence (lower panels) separated. The dotted, white line indicates the border of the wound site. Scale bars $100 \mu \mathrm{m}$.

\section{Figure 2}

$\mathrm{H}_{2} \mathrm{O}_{2}$ treatment rescues regeneration in MEK-inhibited dormant fragments. (A) For each condition, the experimental setup is displayed in the white boxes. Colour code as follows; green: MEK inhibition by PD0325901 (10 $\mu \mathrm{M}, 5$ days), blue: recovery in fresh medium after several washes (1day), red: treatment with $\mathrm{H}_{2} \mathrm{O}_{2}(1,5-2,25 \mathrm{mM}$, 6 hours), yellow: follow up in fresh medium after several washes (7 days). The amputation setup is displayed in the dark grey boxes; $1^{\text {st }}$ cut applied just before MEK-inhibition, $2^{\text {nd }}$ cut applied after recovery period and only applicable in the "REcut" condition). (B) "Dormant", MEK-inhibited, trunk fragments with pharynx (B.1) and tail fragments without pharynx (B.2) were Recut ( $2^{\text {nd }}$ cut) or treated with $\mathrm{H}_{2} \mathrm{O}_{2}$. "NO $\mathrm{H}_{2} \mathrm{O}_{2}$ " controls were neither recut or treated with $\mathrm{H}_{2} \mathrm{O}_{2}$, and were only MEKinhibited. (B.3) Display of the different regenerative outcomes ( 7 days post 
rewounding or $\mathrm{H}_{2} \mathrm{O}_{2}$ treatment) quantified on graphs B.1 and B.2: no regeneration (white), slow regeneration (light blue), normal regeneration (dark blue) and dead (black). "Normal regeneration" is considered as full regeneration including eyes, and if needed pharynx. In the case of "slow regeneration", a clear blastema was visible, however, no eyes and/or pharynx were differentiated at this time point. "No regeneration" refers to the absence of a blastema and a total block of regeneration. Sample numbers are indicated on the right site of each graph. (C) Putative model in which ROS would function upstream of MEK.Scale bars $100 \mu \mathrm{m}$.

\section{Figure 3}

pERK activation decreases after the inhibition of ROS production . (A) The amputation setup is displayed in the grey box, indicating the amputation sites. (B) Immunostaining with an anti-pERK antibody on 1-day regenerating fragments. Animals kept in culture medium or $0,01 \%$ DMSO were used as control animals, while in the treatment group, ROS production was inhibited with DPI ( $3 \mu \mathrm{M})$ administered in the culture medium. All pictures were taken under the same light intensity settings. Sample numbers are indicated in the images. (C) Putative model in which ROS could activate pERK through MEK. Scale bars $100 \mu \mathrm{m}$.

\section{Figure 4}

pERK activation regulation by Smed-egfr-3 andSmed-egr-4. (A) The amputation setup is displayed in the grey box, indicating the amputation sites. Red squares correspond to the anterior blastemas shown in $B$ at 6 hours and 1 day postamputation. (B) Immunostaining with an anti-pERK antibody in controls, after Smedegfr-3 or Smed-egr-4 RNAi. All pictures were taken under the same light intensity settings. In all panels anterior towards the top. Sample numbers are indicated in the images. (C) Putative model in which ERK activation might be regulated by Smedegfr-3 and Smed-egr-4. Scale bars $100 \mu \mathrm{m}$.

\section{Figure 5}

ROS production is regulated by Smed-egfr-3 and Smed-egr-4 during early regeneration. (A) The amputation setup is displayed in the grey box, indicating the amputation sites. The red square corresponds to the images shown in (B) and (D). (B) In vivo visualisation of amputation-induced ROS levels 30 minutes -, 6 hours - 
and 1 day post amputation (MPA, HPA, DPA) in controls and Smed-egfr-3 or Smedegr-4 RNAi fragments. All pictures were taken under the same light intensity settings. Sample numbers are indicated each image. Scale bar 100 $\mu$ m. (C) Putative model showing the possible relationships between ROS, EGFR-3, ERK, egr-4 and mkp. (D) Whole-mount in situ hybridization of Smed-mkp in controls and Smed-egfr-3 or Smed-egr-4 RNAi planarians, 6 hours post-amputation. All panels are oriented with the anterior towards the top. Sample numbers are indicated each image. Scale bar $200 \mu \mathrm{m}$.

\section{Figure 6}

Proposed signaling model of interactions between ROS and the EGFR/MAPK pathway to regulate planarian regeneration. $A$ possible regulatory pathway implicating ROS, egfr-3, egr-4 and MKP is displayed based on literature and the results presented here. .

\section{Supplementary Figure 1}

DPI mediated inhibition of ROS production impairs blastema formation and CNS regeneration. Control animals were kept in culture medium. In an additional control group, animals were exposed to 0,01\% DMSO, while ROS inhibition was mediated by exposure to $3 \mathrm{mM}$ DPI. In the left panels, all images were taken at 3 days post amputation (3DPA). The amputation setup is shown in the central panel. The right panels show regenerating fragments after a immunostaining with antiSYNORF1 in order to visualise the central nervous system at 6 days post amputation (6DPA). Scale bar $200 \mu \mathrm{m}$.

\section{Supplementary Figure 2}

The fluorescent signal at the wound site is ROS production specific and not related to autofluorescence. For each condition, the experimental setup is displayed in the white box; blue: ROS visualisation procedure using carboxy$\mathrm{H}_{2}$ DCFDA, yellow: imaging procedure. Amputation setup is displayed in the light grey box. Regenerative wound (R-wound) \& healing wound ( $\mathrm{H}$-wound) are indicated. All animals were visualised 30 minutes post amputation (MPA). A representative closeup image (merged or separated: bright field and fluorescence) of either an R-wound or $\mathrm{H}$-wound is displayed on the right panel. (A) Control condition with ROS 
visualisation at the site of the R-wound (A.1) and H-wound (A.2). (B) No in vivo ROS stain is performed in order to exclude possible autofluorescence at the wound sites. In both R- (B.1) and H-wound (B.2), no autofluorescence was detected. Because of the absence of a fluorescence signal (B), the close-up of the wound site is shown in bright field (upper panel) and fluorescence (lower panel) separated. The dotted, white line indicates the border of the wound site. Scale bar $100 \mu \mathrm{m}$.

\section{Supplementary Figure 3}

The rescue of regeneration in MEK-inhibited tails by $\mathrm{H}_{2} \mathrm{O}_{2}$ treatment is not attributed to rewounding by the exposure. For each condition, the experimental setup is displayed in the white boxes. Colour code as follows; green: MEK inhibition by PD0325901 (10 $\mu \mathrm{M}, 5$ days), blue: recovery in fresh medium after several washes (1day), red: treatment with $\mathrm{H}_{2} \mathrm{O}_{2}$ (1,5 mM, 3 or 6 hours), grey: ROS visualisation procedure using carboxy- $\mathrm{H}_{2}$ DCFDA, yellow: imaging procedure. The amputation setup is displayed in the grey box; amputation above the pharynx generating a head and tail fragment. $\mathrm{H}_{2} \mathrm{O}_{2}$ treated fragments were in vivo stained and imaged after either 3 - or 6 hours of exposure to $\mathrm{H}_{2} \mathrm{O}_{2}$. A close-up of the epidermis is presented in bright field (left panel) and fluorescence (right panel) separated, and is representable for the whole fragment. The dotted, white line indicates the border of the fragment. (A) control condition: MEK-inhibited fragments without any other intervention. (B) treatment conditions: MEK-inhibited fragments followed by 3- (B.1/2) or 6 hours (B.3/4) of $\mathrm{H}_{2} \mathrm{O}_{2}$-treatment. Scale bar $50 \mu \mathrm{m}$. 


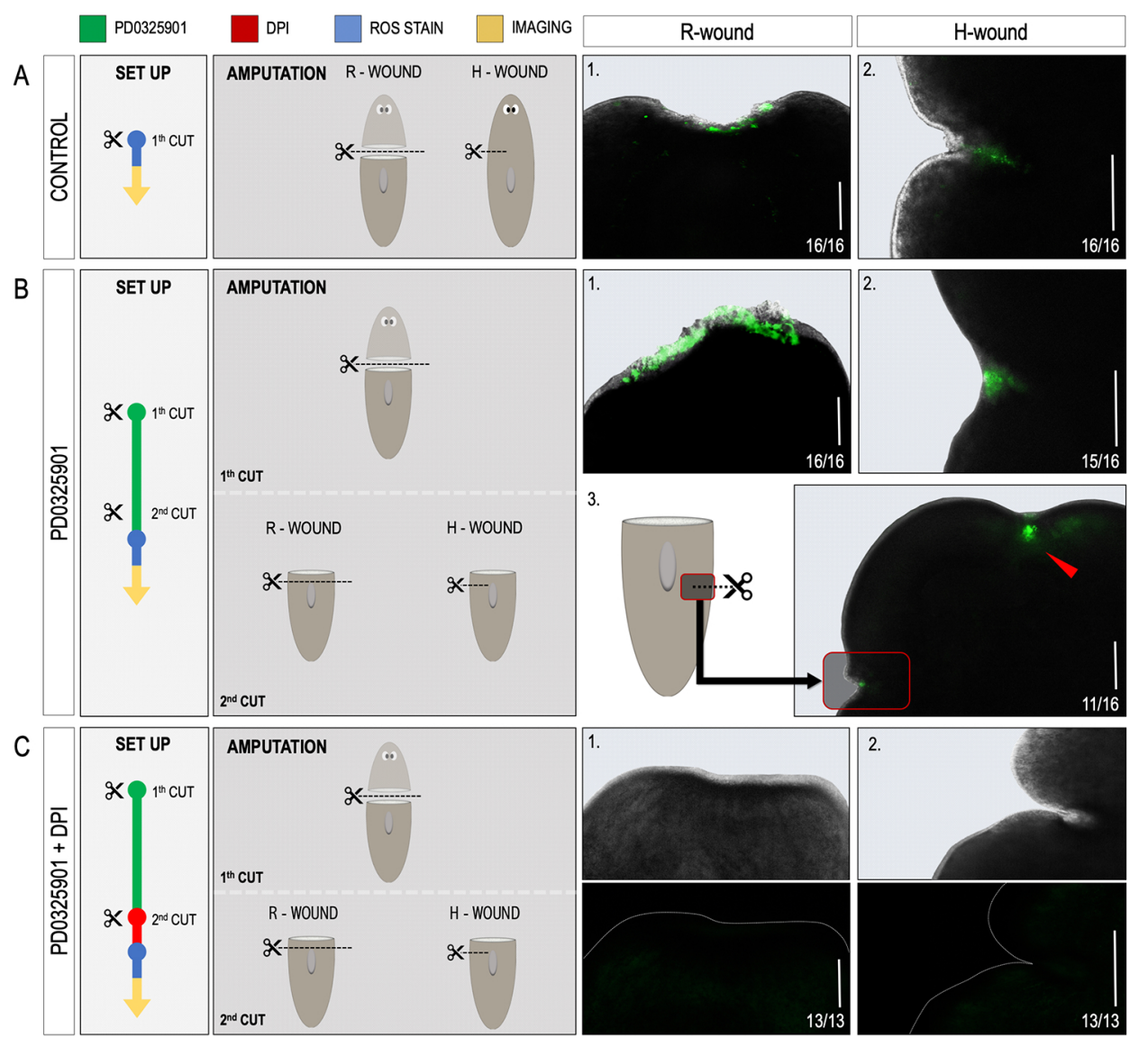




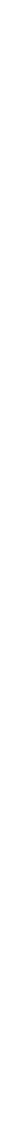




\section{pERK immunostaining | 1DPA}

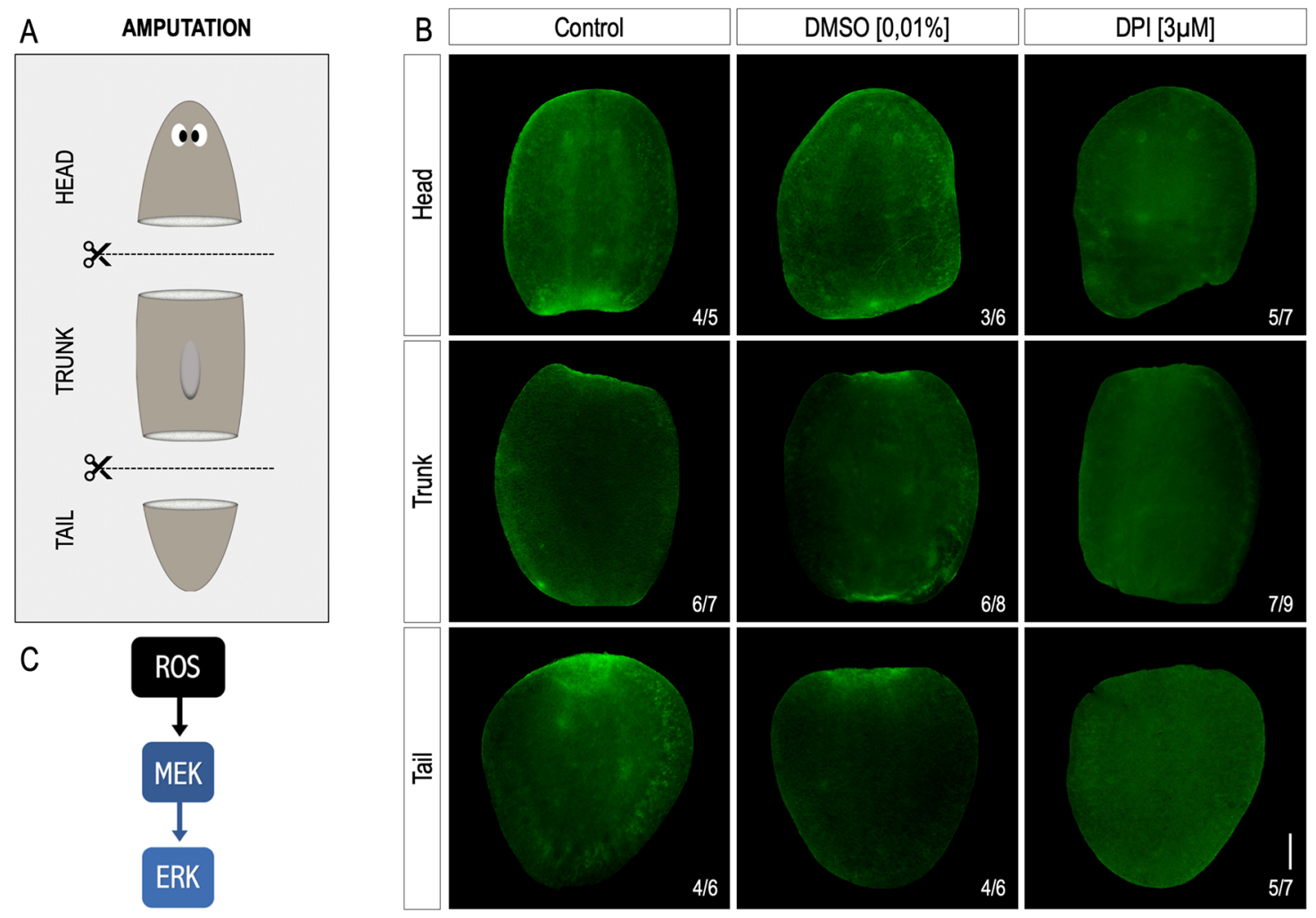


pERK immunostaining

A

AMPUTATION
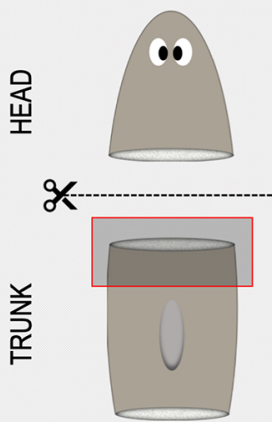

$2 x$

$\bar{E}$

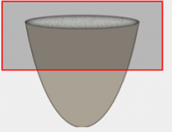

C

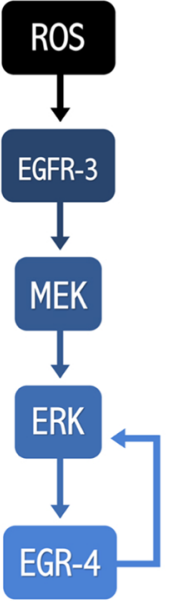

B Trunk

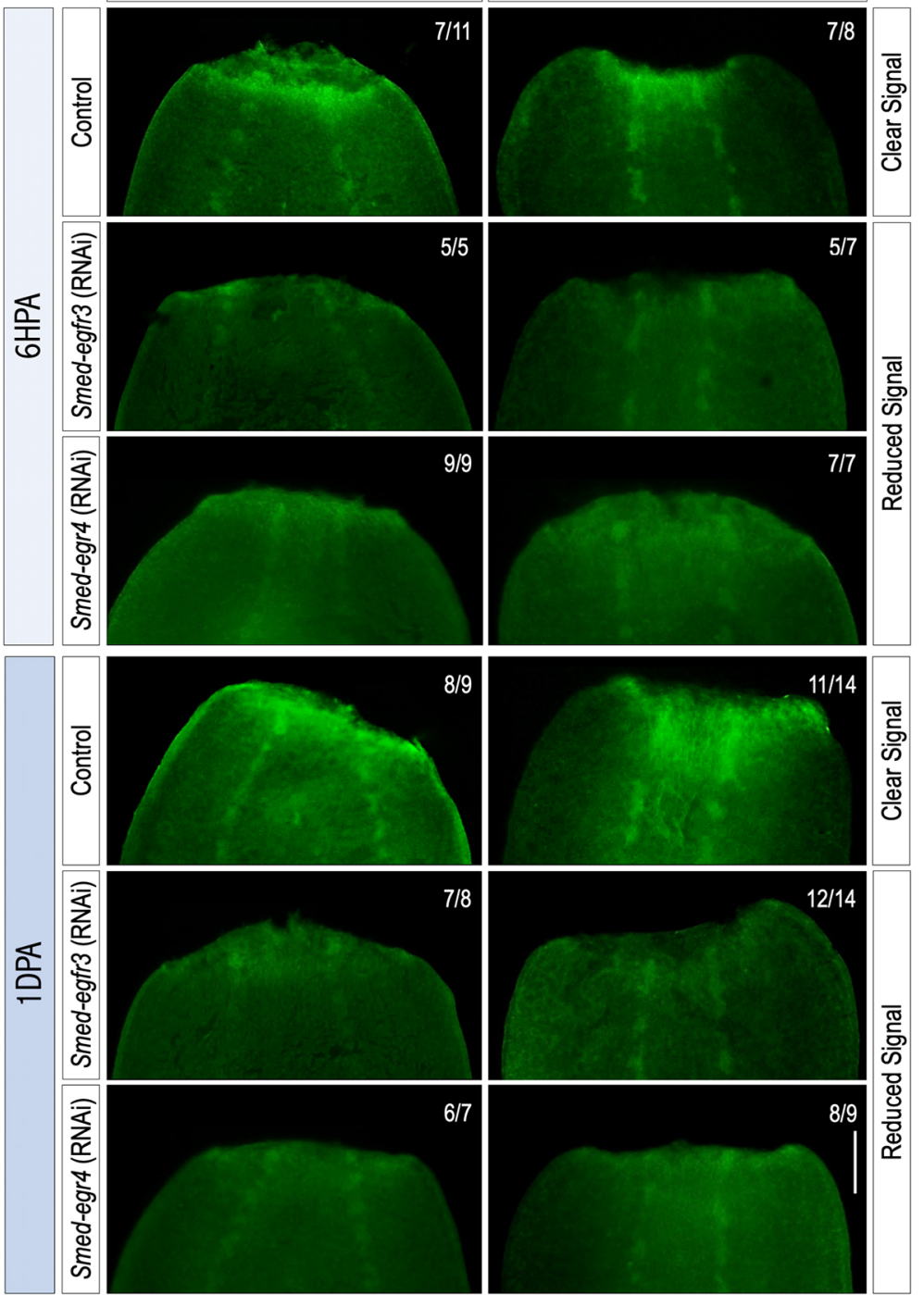


In vivo ROS stain

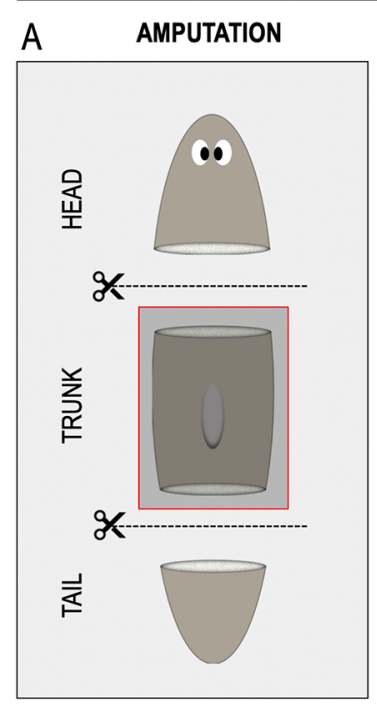

B
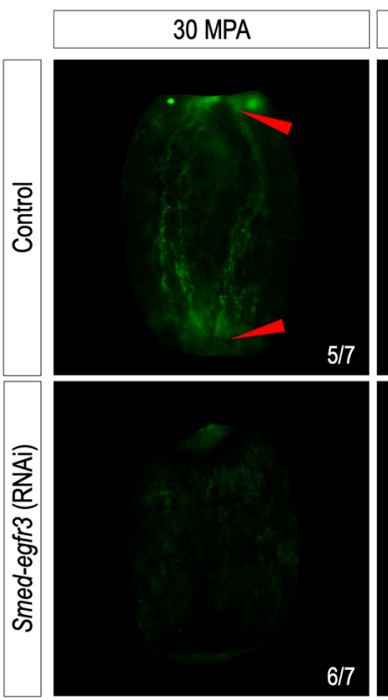

$6 / 7$

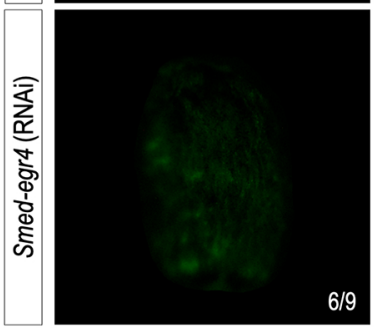

$4 / 7$

$1 \mathrm{DPA}$

$7 / 8$

$6 / 8$

$5 / 7$

$5 / 7$

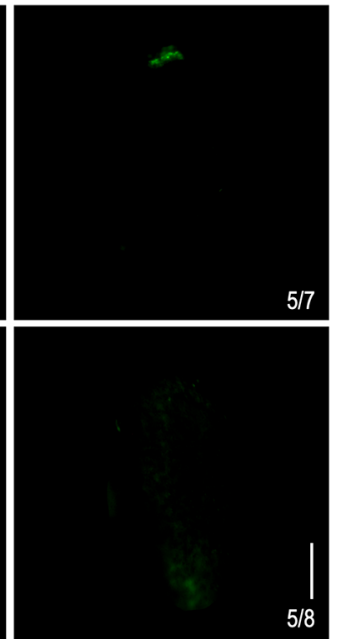

D Whole mount in situ hybridisation - Smed-MKP

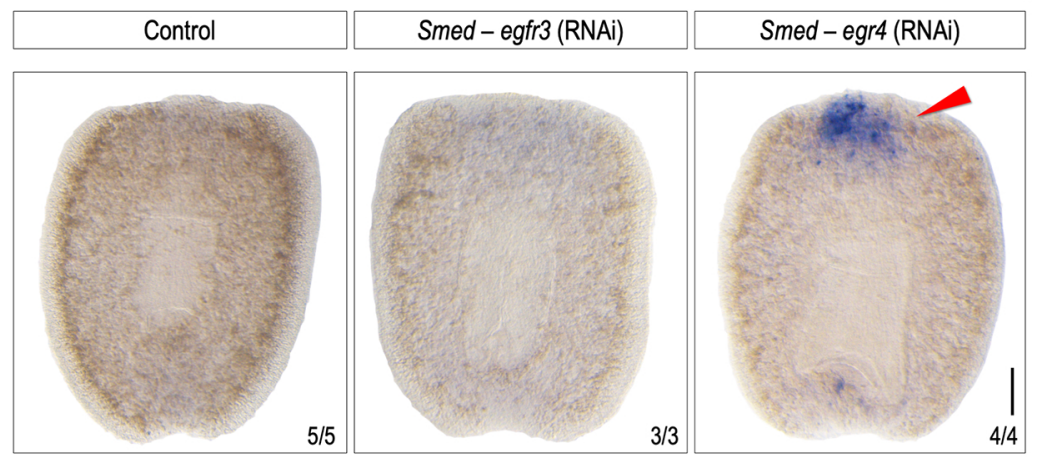


EXTRACELLULAR

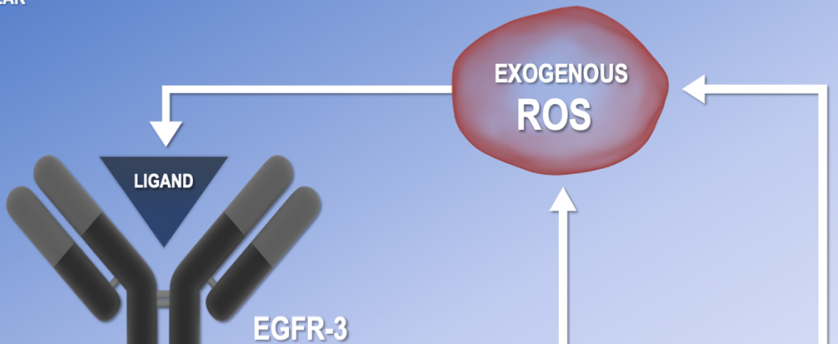

\section{

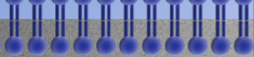

croorasu
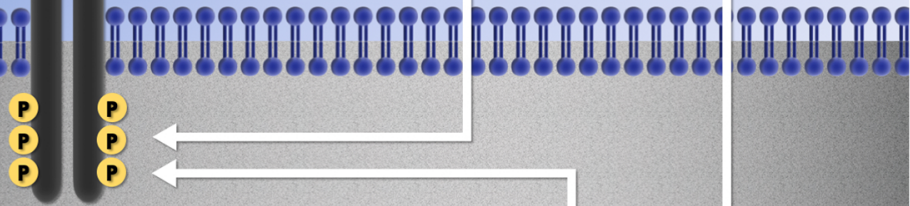


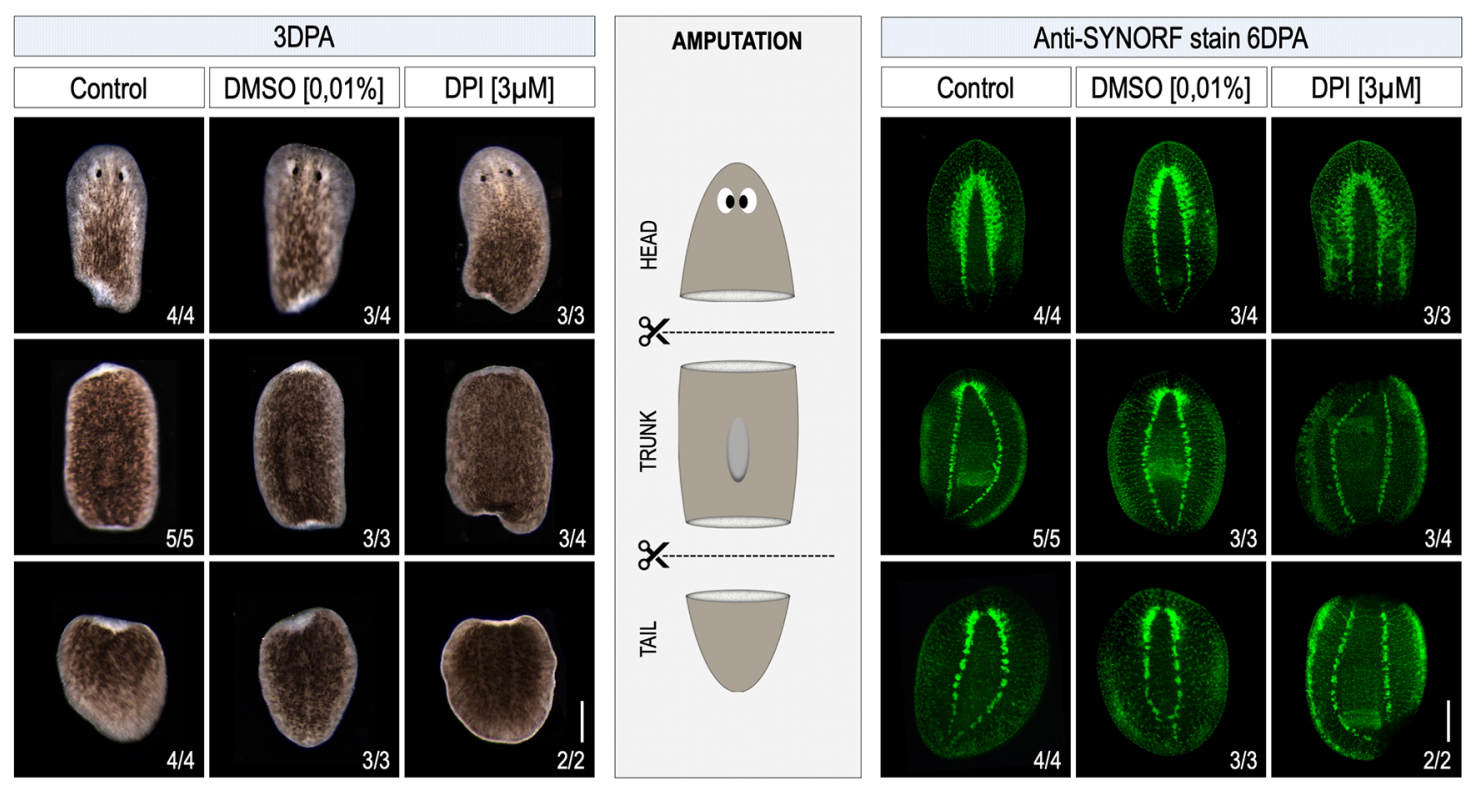




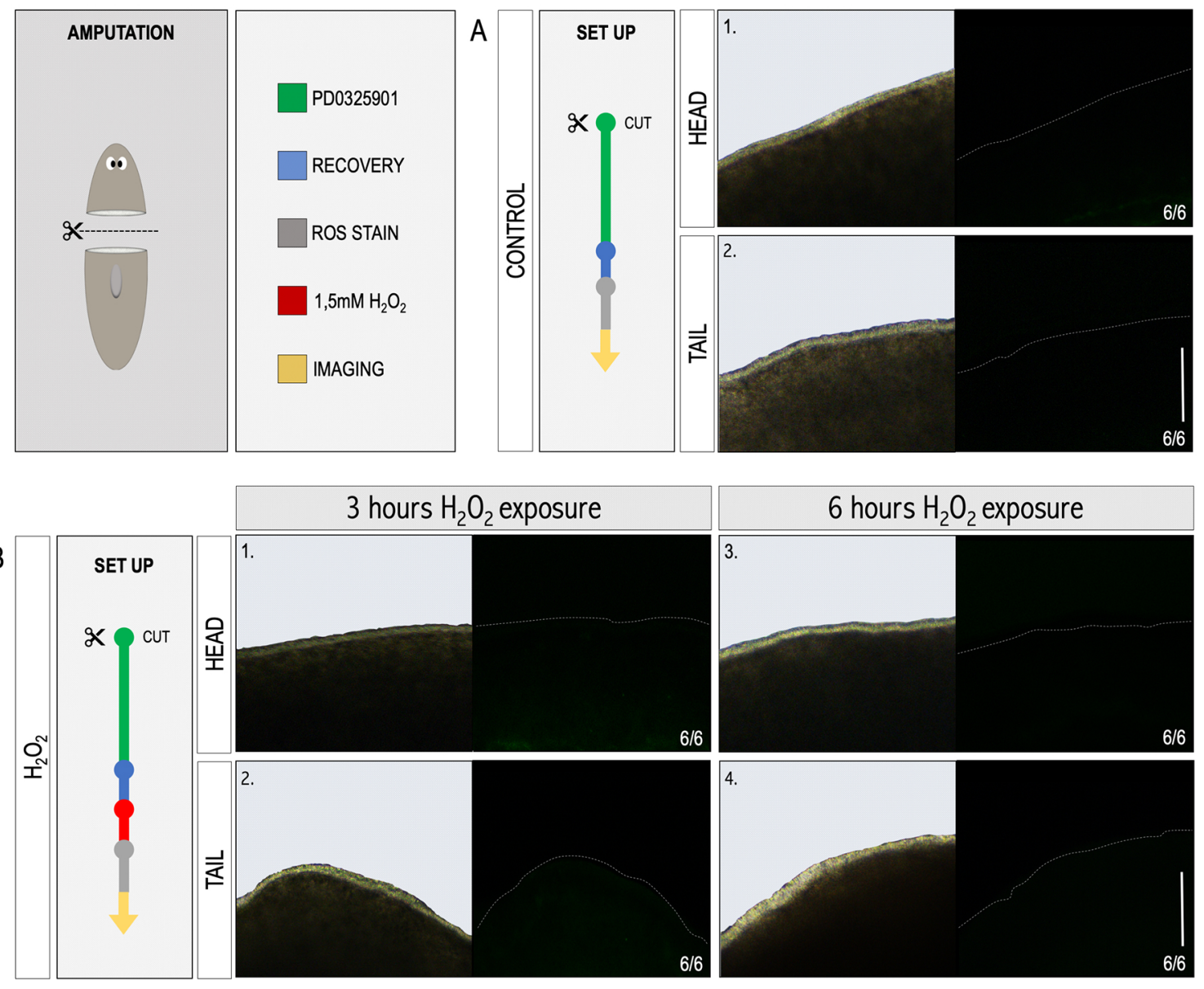

